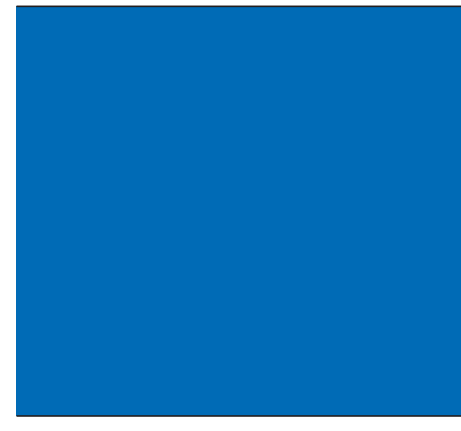
medical community. Based on a shared appreciation of its young but growing scientific foundation, close agreement has been achieved on defining good practice, informed, where possible, by research. This article summarises current international recommendations and the recent changes in thinking that underpin them.

\title{
COMPONENTS OF RESUSCITATION
}

The main components of resuscitation had been established individually by 1960-external chest compression by Kouvenhaven, expired air ("mouth-to-mouth") ventilation by Schafer, and external defibrillation by Zoll; it was also Schafer who first recognised the value of combining these elements into a practical procedure widely suitable for treating collapsed patients.

Cardiopulmonary resuscitation (CPR) is appropriate for a variety of acute medical events where death is likely without immediate intervention. Of these, unheralded ventricular fibrillation has received the most prominent attention partly because of its frequency in patients with ischaemic heart disease, left ventricular failure, and myocardial hypertrophy, and partly because of its unique potential for successful treatment by rapid defibrillation supported by basic life support.'

Other forms of cardiac standstill-asystole and electromechanical dissociation (now called pulseless electrical activity or PEA) — may also be triggered by acute myocardial ischaemia; or they may result from a range of metabolic, toxic or traumatic insults. Asystole and PEA are considerably more resistant to treatment, and recovery is unlikely unless correction can be achieved of an underlying cause such as profound hypoxia, cardiac tamponade, hypovolaemia, hypothermia, drug overdose, electrolyte imbalance, or tension pneumothorax.

In children and younger adults, CPR is required most commonly for respiratory arrest, airway obstruction or drug overdose. Major trauma, external or internal haemorrhage, major pulmonary embolism, profound anaphylaxis, electrocution or a critical cerebrovascular event may call for resuscitation at any age.

The immediate mechanism of a condition requiring resuscitation-as well as any co-existing morbidity or current drug treatment-will determine the optimum approach to emergency treatment. In particular, an unheralded, primary cardiac arrest will cause instant cessation of cardiac output yet with a pool of well oxygenated blood still present in the arterial system; here, rapid restoration of cardiac contraction is the highest priority. ${ }^{2}$ In contrast, airway obstruction or respiratory arrest caused by central depression will have a relatively slow effect in diminishing cardiac output; immediate attention to ventilation becomes the key to cerebral protection, the primary aim of CPR.

\section{KEY STEPS OF MANAGEMENT}

The key steps in any resuscitation attempt comprise:

- avoiding and/or removing danger to both rescuer and patient (the environment can never be assumed to be safe!)

- without delaying urgent treatment, noting the circumstances of the patient's collapse and his/her prior clinical state

- ensuring adequate cerebral and cardiac oxygenation through chest compression and/or positive pressure ventilation; most circumstances dictate that this is achieved initially with no special devices other than a simple facemask (basic life support-BLS)

Correspondence to

Professor Richard Vincent, Postgraduate Medical School Faculty of Health, University of Brighton, Falmer, Brighton BN1 9PH, UK;

R.Vincent@brighton.ac.uk

- applying definitive treatment using special techniques, most commonly defibrillation, intubation, and intravenous cannulation for the administration of drugs and/or fluids (advanced life support-ALS)

- in successful cases, providing post-resuscitation care to maximise cerebral recovery and prevent recurrence of the arrest

- where there is a failure to respond to treatment, judging when the resuscitation attempt should be discontinued. 


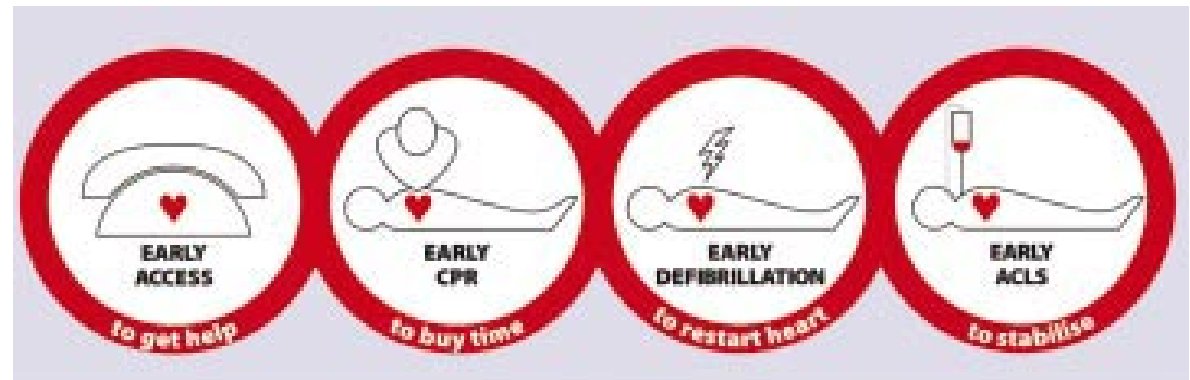

Figure 1 Two representations of the "chain of survival" - a sequence of actions that, when complete and well linked, optimises the chances of survival from cardiac arrest. The key links are "early access", "early CPR", "early defibrillation", and "early advanced care". Upper panel reproduced courtesy of the British Heart Foundation; lower panel reproduced courtesy of the American Heart Association.

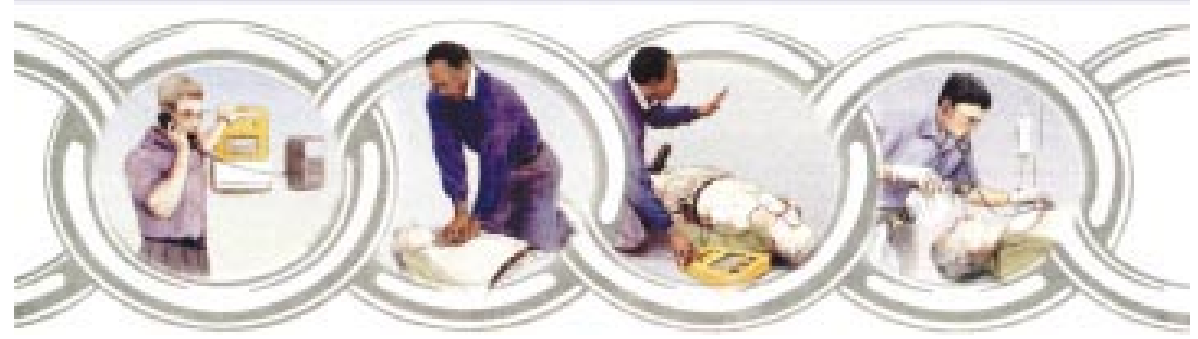

Unless the initial attendant is skilled in all of these steps and has both immediate assistance and the necessary equipment to hand, urgent help will be required. Minimising the delay to effective treatment is mandatory. For each minute lost in the provision of defibrillation to a patient in ventricular fibrillation (VF) the chances of success diminish by $7-10 \%{ }^{3}$ Brain damage occurs within minutes of anoxia and cardiovascular shock rapidly becomes irreversible.

The key steps in delivering CPR are now conveniently represented by the American Heart Association's "chain of survival" (fig 1). For resuscitation to achieve its maximum benefit this chain needs appropriate implementation in hospital, family doctor, ambulance or community settings where fatal or near-fatal events are likely to occur. Clear and effective ownership of this implementation, and of ensuring that each link is sufficiently strong, is critical for success though challenging to provide.

\section{TOWARDS EVIDENCE BASED GUIDELINES}

In the first decade of resuscitation, treatment was empirical, idiosyncratic, and usually led by a member of the junior medical staff without formal training in the required skills. The need became clear to develop standardised, evidence based practice and to establish systems for staff training and practice for its effective delivery. Guidelines were seen as a means not only of encapsulating best practice for most settings but also of achieving two other important goals: removing some of the burden, delay and uncertainty of decision making by the resuscitation team leader; and providing the team with similar expectations of the resuscitation procedure.

Guidelines for the detailed management of CPR have evolved with increasing international collaboration (table 1); they are now among the most carefully formulated and widely agreed in the world. The most recent recommendations determining current clinical practice, with 400 pages of supporting text, were published in March 2000 by an international scientific panel, the International Liaison Committee On Resuscitation (ILCOR). The ILCOR document ${ }^{4}$ combines US, European, Australian, Canadian, South African, and Latin American perspectives.

In their evolution, CPR algorithms for basic and advanced life support have become progressively simpler. The reasons are twofold: continuing scientific evaluation of the drugs previously advocated for CPR has shown many to be ineffective or harmful; and-especially in basic life support-inexperienced helpers appear far less able than first envisaged to remember and carry out the steps of even apparently straightforward actions. In contrast, resuscitation guidelines have also become more comprehensive, dealing with the common prodromes of cardiac arrest (particularly acute coronary syndromes and stroke), arrhythmias likely to pressage or follow an arrest (the 'peri-arrest arrhythmias'), cardiac arrest in a wide range of

Table 1 The historical emergence of guidelines for resuscitation showing progressive collaboration and uniformity

\begin{tabular}{ll}
\hline 1966 & First conference on CPR, NAS-recommended standardised training \\
$1973 / 1974$ & Published AHA guidelines first included CPR training for lay persons \\
$1980 \mathrm{~s}$ & Growth of guidelines for CPR in individual countries \\
1983 & Neonatal and paediatric guidelines published \\
1992 & Landmark agreement for guidelines published in Europe by the ERC \\
1992 & Formation of ILCOR \\
1997 & Advisory statement from ILCOR set first "universal" guidelines in place. Adopted at \\
1998 & once by UK \\
& ERC adopted and published ILCOR advice with minor modifications that reflected \\
& UK experience \\
& First International Guidelines Conference; produced current wide ranging \\
& recommendations with extensive reference to evidence
\end{tabular}

AHA, American Heart Association; CPR , cardiopulmonary resuscitation; ERC, European Resuscitation Council, ILCOR, International Liaison Committee On Resuscitation (see text for membership); NAS, National Academy of Science 


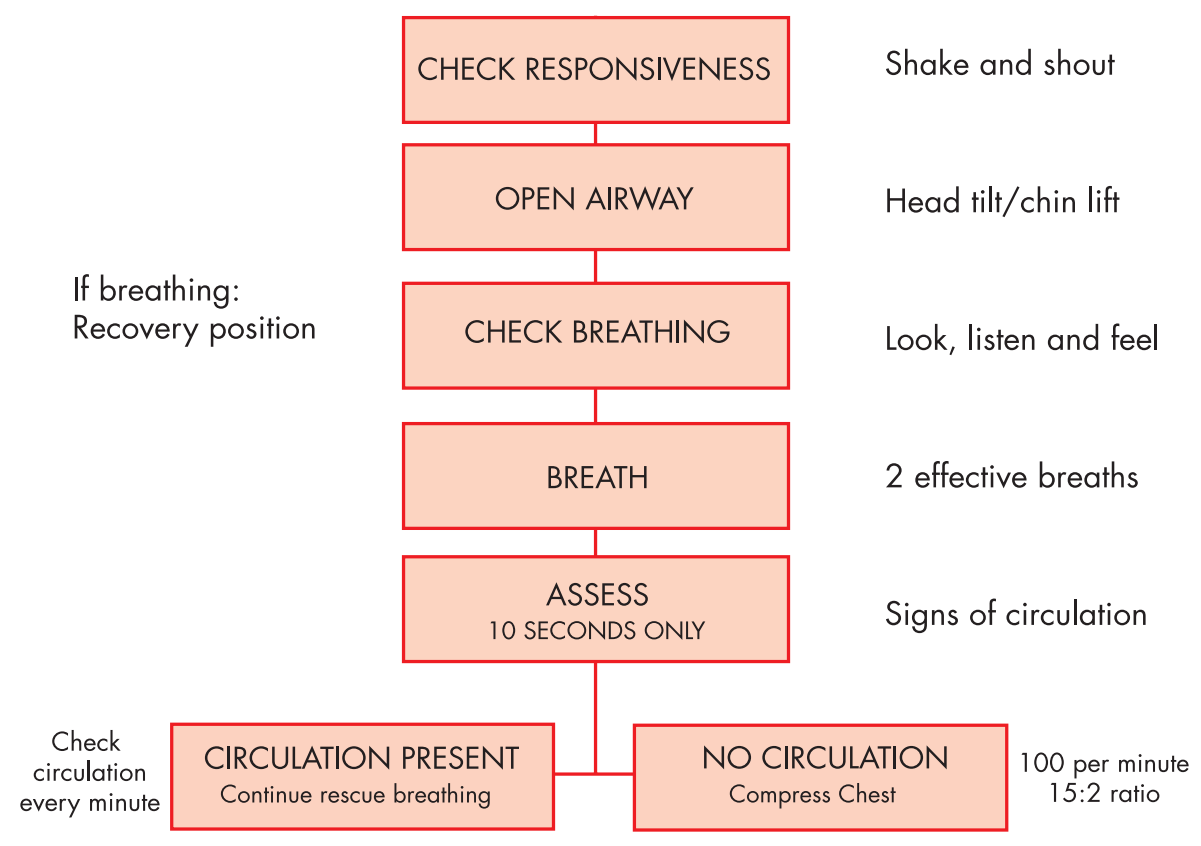

Figure 2 Algorithm for lay persons for adult basic life support following internationally agreed guidelines.

\section{Send or go for help as soon as possible according to the guidelines}

special circumstances, the particular requirements of paediatric and neonatal resuscitation, post-resuscitation care, ethics and legal aspects, and the support of relatives in resuscitation practice.

Guidelines have always provided "best-for-now" advice and their authors recognise the continuing need for scientific data to clarify outstanding questions and misconceptions. But the challenge of acquiring evidence that underpins optimal resuscitation practice should not be underestimated. The pathophysiology of cardiopulmonary arrest is complex, variable, and changes rapidly with time. The effects of drugs and electricity cannot be predicted from a knowledge of their action in nonarrest settings. Studies in patients undergoing resuscitation are fraught with practical and ethical difficulties, and human models of cardiac arrest-for example, in cardiac surgery-are not associated with the complex and threatening metabolic milieu of spontaneous cardiac standstill. Animal experiments have given many helpful clues to pathophysiology and treatment but because of important structural and functional differences, their results cannot be mapped reliably onto the human state.

\section{BASIC LIFE SUPPORT}

At best, basic life support in adults provides 30\% of normal cardiac output, yet this is sufficient to protect the brain and extend the time window for effective defibrillation. ${ }^{5}$ Forward blood flow is achieved as a result of both direct cardiac compression (the "cardiac pump") and by generalised chest compression (the "thoracic pump"). Figures 2 and 3 show the currently recommended sequence. Two versions are provided, one for the lay rescuer with no equipment to hand (fig 2), and one for the health professional who may have ready access to airway adjuncts and a defibrillator (fig 3). In fig 2, checks for responsiveness, airway and breathing are followed in the absence of spontaneous ventilation by giving two effective breaths. Besides helping to correct hypoxia these will provide an additional test of the patient's responsiveness.
Checking for a circulation now deliberately omits feeling for a carotid or femoral pulse. Palpation for major pulses, though formerly the "gold standard" for a diagnosis of cardiac arrest, is time consuming and in many cases misleading; so unresponsiveness, flaccidity, skin colour, and the overall appearance of death are sufficient to indicate full basic CPR in lay (or professional) hands.

During basic life support, a ventilation volume of 700$1000 \mathrm{ml}$ is recommended, consistent with the amount that would ordinarily make the chest rise and fall in a normal fashion. For optimum cardiac output chest compressions should be delivered with a force of about $50 \mathrm{~kg}$ and a rate of 100 per minute - and the ratio of compression to ventilation of 15:2 should be maintained no matter how many rescuers are involved. Coronary blood flow during resuscitation-a critical determinant of recovery-is significantly higher with rapid compressions and minimum interruption of chest compression. Recent studies have shown that a ratio of 5:1 may allow effective chest compression for only $33 \%$ of the time, causing an important loss in cardiac output. ${ }^{7}$ Extending this concept further into clinical practice by omitting all ventilation early in a resuscitation attempt is currently under review. ${ }^{28}$

Health professionals with immediate facilities for airway management and defibrillation are advised to make a rapid, near simultaneous assessment of responsiveness, pulse, and breathing (fig 3). Unless a pulse is definitely present (with no more than 10 seconds allowed for assessment) the application of a monitor/defibrillator is the next step to achieve defibrillation as necessary with minimum delay. As in all resuscitation guidelines, the need is emphasised to seek expert help urgently - at the first positive confirmation of collapse.

In unconscious patients in whom spontaneous ventilation is preserved, airway protection can readily be afforded by a simple oropharyngeal or Guedel airway. A nasopharyngeal airway is better tolerated in patients who are not deeply unconscious and may be essential in the presence of maxillofacial injuries, though it is harder to use and carries a greater risk of complications. A standard oxygen mask with a high 


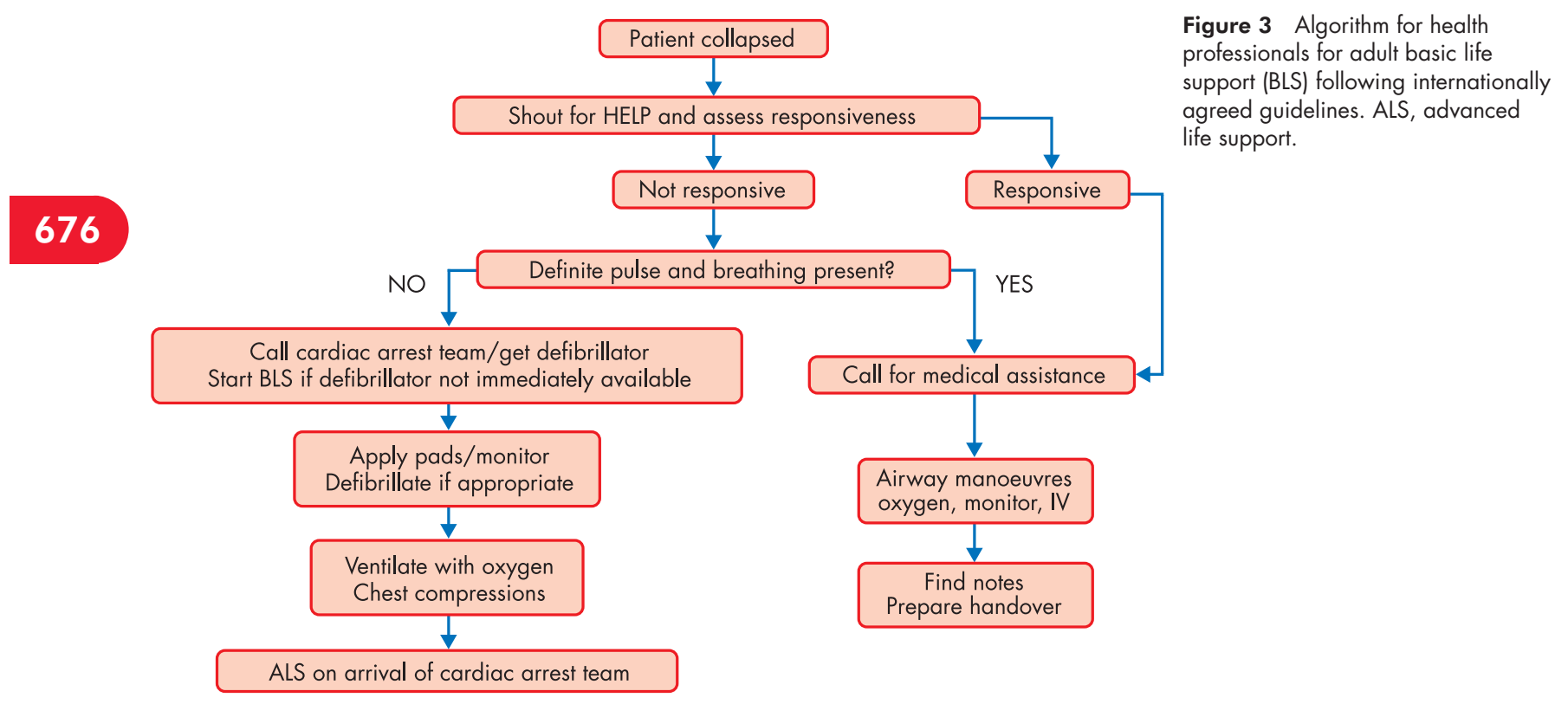

flow of oxygen will deliver a concentration of about 50\%; but in resuscitation, a mask with a reservoir bag is preferable giving concentrations of $85 \%$ with oxygen flows of 10-15 litres per minute $(1 / \mathrm{min})$. The techniques for positive pressure ventilation are described below.

\section{ADVANCED LIFE SUPPORT}

Figure 4 shows the "universal", internationally agreed algorithm for advanced life support (ALS) in adults, the current basis for practice in many countries. Its emphasis is on immediate defibrillation for VF or pulseless ventricular tachycardia (VT). The precordial thump is retained "if appropriate"-meaning a single attempt in cases of a witnessed or monitored arrest by a health professional before the defibrillator is attached. It is unlikely to be effective beyond 30 seconds from the onset of VT or VF. Basic life support should be no more than a short lived bridge to more definitive treatment and should not interrupt the initiation of defibrillation. $^{2}$

\section{Defibrillation}

The diagnosis of either VF/pulseless VT or non-VF/VT (essentially asystole and PEA) determines the next steps in the ALS sequence. For VF/pulseless VT, manual defibrillation with up to three consecutive shocks should be administered within one minute (made possible by short defibrillator recharging times). Checking the pulse after a shock is advised only if it has resulted in a rhythm compatible with a cardiac output. During the initial three shock sequence, no basic CPR is needed. If an automated external defibrillator (AED) is used, its signal and voice prompts should be followed while attempting defibrillation with up to three consecutive shocks. Thereafter, if sinus rhythm has not been restored, CPR should be reinstated for one minute before further defibrillation is attempted.

For conventional defibrillators using a damped sinusoidal monophasic waveform, no change has been made in the initial energy sequence: $200 \mathrm{~J}, 200 \mathrm{~J}, 360 \mathrm{~J}$. Biphasic waveforms may increase the efficacy of defibrillation at lower energies and may avoid the need for escalating the delivered shock. Several potential advantages ensue: reduced myocardial stunning, improved safety, and the use of simpler, lighter electronic components. Biphasic shock defibrillators are becoming

increasingly available, but their optimum characteristics and use, and their long term advantages over monophasic devices, have yet to be determined. ${ }^{9}$

The efficacy and safety of defibrillation is improved by good electrode contact and correct positioning. Gel pads and firm pressure (about $10 \mathrm{~kg}$ force) improve patient contact for manually applied paddles. The adhesive electrodes used with an AED carry the additional advantage of conforming well to the chest contour.

\section{Airway and artificial ventilation}

Failure of defibrillation, or the presence of a non-shockable rhythm, invites airway support and the delivery of optimum ventilation. Positive pressure requires at least a well fitting face mask combined with a simple device for maintaining a patent airway. Inflation can be provided by mouth-to-mask ventilation (preferably with enhanced oxygen delivery) or by a bag-valve device with the following characteristics: selfrefilling, inclusion of a true non-rebreathing valve, and capacity for a maximum oxygen inlet of $30 \mathrm{l} / \mathrm{min}$. But enthusiastic use of this device can result in overventilation with gastric inflation, regurgitation, and pulmonary aspiration. For now mandatory infection control, masks should be disposable for single use or sterilised before re-use.

The use of a face mask, however, is only a holding procedure until either spontaneous recovery occurs or the expertise and equipment are available for the ventilation technique of choice-tracheal intubation. An endotracheal tube affords good airway patency, allows the suction of secretions, ensures a high delivery of oxygen, and protects the airway from aspiration. Ideally, correct tube placement should be confirmed by clinical assessment supplemented by the measurement of arterial blood gasses and end tidal carbon dioxiode. Endotracheal delivery through the tube of oxygen enriched air, either by the bag-valve apparatus or by an automatic ventilator, should provide a tidal volume of about $6-7 \mathrm{ml} / \mathrm{kg}$ at $10-12$ breaths/min.

Intubation requires training and repeated practice to maintain competence. Alternative airways are available that can be inserted without direct visualisation of the upper airway and may therefore be appropriate when the endotracheal route has failed or the expertise or equipment for its use is not to hand. 


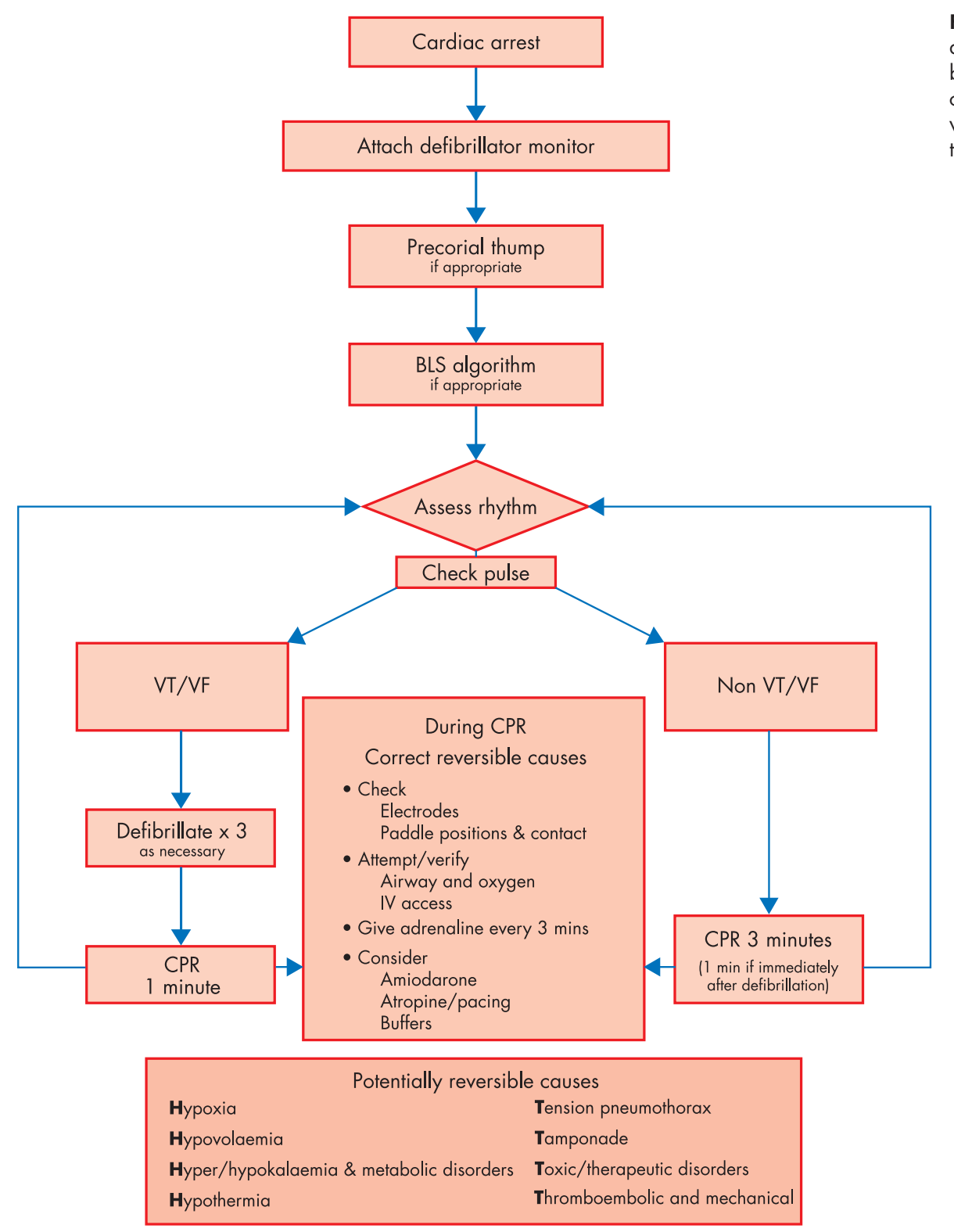

Figure 4 "Universal" algorithm for adult advanced life support. BLS, basic life support; CPR, cardiopulmonary resuscitation; VF, ventricular fibrillation; $\mathrm{VT}$, ventricular tachycardia.

The laryngeal mask airway and the double lumen oesophageal-tracheal "Combitube" are designed for "blind" insertion; after appropriate inflation of their cuffs they afford airway protection and a more secure system for oxygen delivery than the bag-valve-mask combination. Though seemingly easier to use than an endotracheal tube, however, correct use of these devices requires practice and vigilance, and important complications can arise. ${ }^{10}$

\section{Circulatory support}

Chest compressions at a rate of 100 per minute should be uninterrupted except for brief pulse checks and for defibrillation; a pause of over 15 seconds threatens the success of CPR and reduces myocardial function in the post-arrest period. There is no need for synchrony between compressions and ventilation. Operator fatigue threatens the efficiency of chest compression with a consequent reduction in cardiac output after a surprisingly short time (table 2).
New adjuncts to CPR have been explored for increasing cardiac output during resuscitation. ${ }^{11}$ In interposed abdominal compression pressure of about $100 \mathrm{~mm} \mathrm{Hg}$ is applied rhythmically to the abdomen half way between the xiphisternum and umbilicus by a second rescuer during the relaxation phase of cardiac compression. This "abdominal pump" mechanism for haemodynamic augmentation appears from small trials to improve the return of spontaneous circulation (ROSC) and 24 hour survival, particularly of patients treated in hospital. It may also reduce the rate of gastric inflation before tracheal intubation.

Active compression-decompression devices allow application of a negative intrathoracic pressure alternately with positive chest compression during CPR. Experimentally they produce improved arterial pressure and vital organ perfusionbut they show no unequivocal benefit in patient outcome and are not entirely straightforward to use. Mechanical CPR by 
Table 2 The deteriorating performance of chest compressions with time in a group of 40 adult subjects

\begin{tabular}{lll}
\hline Minutes of compression & Total compressions per minute & $\begin{array}{l}\text { Number of satisfactory } \\
\text { compressions }\end{array}$ \\
\hline First & 108 & 82 \\
Second & 105 & 68 \\
Third & 105 & 52 \\
$*$ & 110 & 70 \\
Fourth & 105 & 44 \\
Fifth & 103 & 27 \\
Sixth & & \\
\hline
\end{tabular}

*A 30 second rest was given to the subjects after the third minute.

Data from Ashton A, McClusky A, Gwinnutt CL, et al. Effect of rescuer fatigue on performance of continuous

external chest compressions over 3 minutes. Resuscitation 2002;55:151-5.

devices that rhythmically depress the sternum or circumferentially compress the chest offer relief to rescuers in prolonged resuscitation and may allow simultaneous chest compression and defibrillation, but they have not yet been shown to provide any additional long term patient benefit.

\section{Drugs}

Since resuscitation began drugs have been included for emergency life support. The use of most agents began as an extrapolation from their effects in clinical circumstances other than cardiopulmonary arrest. Most were chosen empirically to correct obvious clinical findings or assumed pathological mechanisms: any of a variety of antiarrhythmic agents to terminate VT, enhance defibrillation or prevent further major arrhythmias; "stimulants" such as adrenaline (epinephrine), calcium, or atropine to increase cardiac rate or contractility; sodium bicarbonate to oppose acidosis in the face of poor ventilation and perfusion. But with a growing scientific basis for resuscitation it became clear that in the highly disordered pathophysiology of the arrested patient, drug distribution and effect could not readily be predicted, that each agent in current use lacked any evidence for its benefit, and that most were capable of exerting important effects adverse to recovery.

The number of pharmacological agents now recommended for resuscitation is small. Space prevents a detailed discussion of the rationale for their use but this is readily accessed elsewhere. $^{412} 13$

Adrenaline (epinephrine) $1 \mathrm{mg}$ after every cycle of three unsuccessful shocks or after every three minutes of CPR during a non-shockable arrest improves cerebral and coronary blood flow. Experimentally-mostly in animals-it increases peripheral resistance by $\alpha$ adrenergic stimulation, thereby preventing arterial collapse during the release phase of cardiac compression. It may also increase myocardial contractility and rate by $\beta$ adrenergic stimulation after restoration of an effective heartbeat, or if in apparent PEA cardiac contraction is present but impalpable. Perhaps surprisingly, its benefit for survival in man is still debatable; no randomised controlled trial has been attempted to support its use (and probably never will be). High dose adrenaline has no clear advantage and may be deleterious. ${ }^{14}$

Vasopressin may be an acceptable alternative to adrenaline to support cardiovascular collapse, ${ }^{15}$ although evidence confirming the value of its use is still being gathered.

Amiodarone given initially as a $\mathbf{3 0 0} \mathrm{mg}$ bolus is the first line recommendation for shock refractory VF/VT. The ARREST trial in 504 patients refractory to three shocks for out-of-hospital VF reported improved survival to hospital admission compared with placebo $(44 \% v 34 \%) .{ }^{16}$ But survival to discharge was identical in each group at $13 \%$.
Lignocaine is an alternative to amiodarone in the universal algorithm for the management of cardiac arrest, but has not been adopted by the European Resuscitation Council. In the CALIBRE study comparing lignocaine and bretylium for refractory $\mathrm{VF}^{17}$ neither showed any benefit against placebo in promoting ROSC.

Magnesium is suggested for refractory VT/VF if the patient could be magnesium or potassium depleted, although support for its use is predominantly anecdotal.

Atropine in a single bolus of $3 \mathrm{mg}$ is recommended for PEA when the heart rate is less than 60 beats/min; but the window for achieving benefit by reducing vagal tone during the course of cardiac arrest is small.

Buffer agents (mainly sodium bicarbonate) are relegated to late in the management of an arrest. The correction of acid-base balance during resuscitation depends primarily on adequate alveolar ventilation. No data confirm that treatment with buffer agents improves outcome; in contrast, intravenous bicarbonate may produce intracellular acidosis that damages cardiac or cerebral tissue. Buffers may be appropriate in patients very late in the arrest procedure or in those with known pre-existing metabolic acidosis, hyperkalaemia, or tricyclic overdose. Full blood gas analysis should always guide their use.

Calcium salts have been abandoned since evidence for their efficacy is lacking while data point to their adverse effect on cellular survival after reperfusion.

All drugs during an arrest are best given through intravenous access sited as centrally as possible; all other routes are unreliable.

Drugs also feature prominently in the new CPR algorithms for the "peri-arrest arrhythmias", rhythm disorders that may precede or follow an arrest and are worthy of treatment in their own right. Separate algorithms cover narrow-complex tachycardias, broad-complex tachycardias, bradycardias, and atrial fibrillation. But here too extrapolation, anecdote, and fashion are more readily available than scientific evidence for the choice of therapeutic agent.

\section{ORGANISATION AND TRAINING}

Effective implementation of CPR guidelines in hospital depends primarily on strong ownership, audit and supervision of the resuscitation service, and its clear integration into local operational and governance policies. Without this, resources and encouragement will be insufficient to sustain adequate training in personal skills and teamwork and the readiness of strategically placed equipment. To speed the response to VF, the use of AEDs and the deployment of nurse defibrillation is effective and should be explored. ${ }^{2}{ }^{18}$ 


\section{New features in current resuscitation practice}

OUT

- Any palpation for a pulse before starting cardiopulmonary resuscitation (CPR) (lay rescuer)

- Palpation for pulse after a DC shock unless a coordinated rhythm is present

- Most previously used drugs, except adrenaline (epinephrine)

- High dose adrenaline

IN

- Concern about fatigue during CPR

- Strategies for maintaining coronary blood flow during an arrest

- Amiodarone as first line treatment for refractory ventricular fibrillation (VF)

- New, widely agreed algorithms including those for peri-arrest arrhythmias

- Widespread use of automated external defibrillators (AEDs) to speed the response to VF

- The continued need for training

PROMISING

- Vasopressin for circulatory support

- Biphasic waveforms for defibrillation

- Nurse led defibrillation

- Hospital based medical emergency teams (MET)

- Recent mechanical devices for chest compression

- Interposed abdominal compression

- Cooling for cerebral protection

Deciding who should not receive attempted resuscitation is an important and sensitive issue, one that should be engaged thoroughly by the consultant and the caring team in every case. The statement published jointly by the British Medical Association, the Resuscitation Council (UK), and the Royal College of Nursing on "Decisions relating to cardiopulmonary resuscitation" ${ }^{\prime 19}$ is essential reading.

Outside hospital the success of resuscitation remains disappointingly poor, generally well under $10 \%,{ }^{1}$ yet this should encourage rather than deter the further training of both health professionals and lay persons in the community. Paramedic services are well established although their effectiveness is inevitably affected by local geography and detailed operational arrangements. Immediate resuscitation by primary care or other community health staff, and by lay rescuers, enhances patient survival particularly through the advent of the AED. ${ }^{20-22}$ Unwarranted fears of infection or litigation should not deter the attempted rescue of a patient whose only outcome without intervention will be death. ${ }^{23}$

\section{WHERE NEXT?}

Two therapeutic challenges during cardiac arrest remain prominent-maximising coronary perfusion and providing effective cerebral protection. The former results in greater resuscitation success and better long term myocardial preservation. Where patients in VF are seen within four minutes of their collapse, immediate defibrillation with simultaneous chest compression may prove ideal-afforded by developments in AED and mechanical CPR technology. After this, where the delay to defibrillation is more than four minutes (or is unknown), evidence is growing that 90 seconds of CPR before a DC shock may improve outcome. ${ }^{24}$

Cerebral protection is harder; drugs are as unlikely to help here as they are in supporting the heart, for reasons at least of absorption and distribution. Local or systemic cooling could prove practical and worthwhile and warrants further investigation. ${ }^{25}$

Finally, an important and growing theme in resuscitation is that of preventing rather than treating a medical catastrophe. A pattern of deterioration in the hours or days before a cardiopulmonary arrest is common. ${ }^{26}$ Hospital based medical emergency teams (MET) are now emerging, staffed with senior nurses and doctors skilled in the care of the critically ill to seek out, assess, and manage patients whose clinical state threatens impending cardiac arrest. ${ }^{27}$ Precautionary intervention and the wise selection of patients in whom resuscitation will not be appropriate seem likely to improve substantially the success rate of resuscitation attempts.

\section{REFERENCES}

1 Herlitz J, Bång A, Gunnarsson J, et al. Factors associated with survival to hospital discharge among patients hospitalised alive after out of hospital cardiac arrest: change in outcome over 20 years in the community of Göteborg, Sweden. Heart 2003;89:25-30.

- An instructive if rather depressing report of the lack of improvement in survival of patients sustaining an out-of-hospital arrest over a $\mathbf{2 0}$ year period with an analysis of the factors associated with recovery.

2 Spearpoint KG, McLean C, Ziderman D. Early defibrillation and the chain of survival in 'in-hospital' adult cardiac arrest; minutes count. Resuscitation 2000;44: 165-9.

3 Cummins RO. From concept to standard of care? Review of the clinical experience with automated external defibrillators. Ann Emerg Med 1989:18:1269-75

4 American Heart Association, in collaboration with the International Liaison Committee on Resuscitation and Emergency Cardiovascular Care. An international consensus on science. Resuscitation 2000;46:1-448.

- The definitive and most recent presentation of current ILCOR guidelines for resuscitation supported by an extensive review of their underpinning science.

5 Waalewijn RA, Nijpels MA, Tijssen JG, et al. Prevention of deterioration of ventricular fibrillation by basic life support during out-of-hospital cardiac arrest. Resuscitation 2002;54:31-6.

6 Bahr J, Klinger H, Panzer W, et al. Skills of lay people in checking the carotid pulse. Resuscitation 1997;35:23-6

7 Babbs CF, Kern KB. Optimum compression to ventilation ratios in CPR under realisitic, practical conditions: a physiological and mathematical analysis. Resuscitation 2002:54:147-57.

- A persuasive study in favour of maximising the rate of chest compressions to optimise cardiac output and coronary perfusion during cardiac arrest.

8 Hallstrom A, Cobb L, Johnson E, et al. Cardiopulmonary resuscitation by chest compression alone or with mouth-to-mouth ventilation. N Engl J Med 2000;342:1546-53.

9 Marlens PR, Russell JK, Wolcke B, et al. Optimal response to cardiac arrest study: defibrillation waveform effects. Resuscitation 2001:49:233-43.

10 Rumball CJ, Macdonald D. The PTL, Combitube, laryngeal mask, and oral airway: a randomized prehospital comparative study of ventilatory device effectiveness and cost-effectiveness in 470 cases of cardiorespiratory arrest. Prehosp Emerg Care 1997;1:1-10.

11 Wik L. Automatic and mechanical external chest compression devices for cardiac pulmonary resuscitation. Resuscitation 2000;47:7-25.

- A well referenced and highly illustrated review; not entirely up to date, but very instructive.

12 Colquhoun MC. Drugs and their delivery (chapter 17). In: Colquhoun $M C$, Handley AJ, Evans TR, eds. ABC of resuscitation, 4th ed. London: BM Books, 1999:73-6.

13 Vincent R. Drugs in modern resuscitation. Br J Anaesth 1997;79:188-97

- Less "modern" now, but summarises the key information on which most decisions have been made concerning the use of drugs during resuscitation.

14 Vandycke C, Martens P. High dose versus standard dose epinephrine in cardiac arrest - a meta analysis. Resuscitation 2000;45: 161-72.

15 Stiell IG, Hebert PC, Wells GA, at al. Vasopressin versus epinephrine for inhospital cardiac arrest: a randomised controlled trial. Lancet 2001;358:105-9.

16 Kudenchuck PJ, Cobb LA, Copoass MK, et al. Amiodarone for resuscitation after out-of-hospital cardiac arrest due to ventricular fibrillation. N Engl J Med 1999;341:871-8.

17 Tunstall-Pedoe H, Woodward M, Chamberlain DA. Lignocaine and bretylium in resistant ventricular fibrillation: results of the CALIBRE randomised controlled trial. Eur Heart J 2001;22 (abstract suppl):P2368:449

18 Kenward C, Castle N, Hodgetts TJ. Should ward nurses be using automatic external defibrillators as first responders to improve the outcome from cardiac arrest? A systematic review of the primary research. Resuscitation 2002;52:31-8. 
- A careful review indicating the potential value of nurse defibrillation that many have seen but which, in the authors' view, needs substantiating with further research.

19 British Medical Association, the Resuscitation Council (UK) and the Royal College of Nursing. Decisions relating to cardiopulmonary resuscitation. A joint statement from the British Medical Association, the Resuscitation Council (UK) and the Royal College of Nursing. London: BMA, 2001

- Mandatory reading - and an essential area to engage in for al looking after patients in hospital.

68020 Moule P, Albarran JW. Automated external defibrillation as part of BLS: implications for education and practice. Resuscitation 2002;54:223-30.

21 Colquhoun MC. Defibrillation by general practitioners. Resuscitation 2002;52:143-8.

- A key paper by a practitioner/observer with a unique engagement in this area.

22 Monsieurs KG, Handley AJ, Bossaert LL. European Resuscitation Council guidelines for 2000 for automated external defibrillators. A statement from the BLS/AED working group approved by the executive committee of the European Resuscitation Council. Resuscitation 2001;48:201-9.

- ERC guidelines for the use of AEDs. Fundamental reading.

23 Eisenburger $\mathbf{P}$, Sofar $\mathbf{P}$. Life supporting first aid training of the public review and recommendation. Resuscitation 1999;41:3-18.
24 Cobb LA, Farenbuch CE, Walsh TR, et al. Influence of cardiopulmonary resuscitation prior to defibrillation in patients with out of hospital defibrillation. JAMA 1999;281:1182-8.

25 Bernard S, Buist $M$, Montiero $O$, et al. Induced hypothermia using large volume, ice-cold intravenous fluid in comatose survivors of out-of-hospital cardiac arrest: a preliminary report. Resuscitation 2003;56:9-13.

26 Franklin C, Matthew J. Developing strategies to prevent in-hospital cardiac arrest: analyzing responses of physicians and nurses in the hours before the event. Crit Care Med 1994;22:244-7.

27 Hodgetts TJ, Kenward G, Vlackonikolis I, et al. The identification of risk factors for cardiac arrest and formulation of activation criteria to alert a medical emergency team. Resuscitation 2002;54:125-31.

- One of a pair of papers that articulate a clear case for prevention and the formation of in-hospital teams for achieving it.

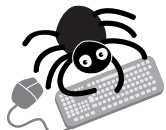

Additional references appear on the Heart website - www.heartinl.com/supplemental 